\title{
Implementation of the Huygens Absorbing Boundary Condition in Corner Regions
}

\author{
Fumie Costen, Member, IEEE, and Jean-Pierre Bérenger, Fellow, IEEE
}

Abstract-In recent years, three new absorbing boundary conditions (ABCs) have appeared in the literature, namely, the multiple absorbing surfaces, the reradiating boundary condition, and the Huygens $\mathrm{ABC}(\mathrm{HABC})$. The last is a generalization of the first two. The $\mathrm{HABC}$ mainly relies on the radiation of a field opposite to the outgoing field by means of surface currents. This paper focuses on the implementation of the $\mathrm{HABC}$ in the corner regions of computational domains. It is shown rigorously that the Huygens surface radiating the opposite field is not a normal Shelkunoff surface. Additional branches, called extensions, must be added in the corner regions.
\end{abstract}

Index Terms-Absorbing boundary condition (ABC), finite difference, finite-difference time-domain (FDTD) method, Huygens surface.

$\mathbf{T}$ WO novel absorbing boundary conditions (ABCs) were presented independently some years ago in the literature: the multiple absorbing surfaces [1] and the reradiating boundary condition (rRBC) [2], [3]. Both rely on the same principle of canceling the outgoing field leaving a computational domain by means of equivalent currents that radiate a field equal in magnitude and opposite in sign to the field to be cancelled. This concept has been generalized and investigated in details in [4], where it is called the Huygens ABC (HABC).

As shown in [4], the $\mathrm{HABC}$ is equivalent to a traditional operator ABC. This is because the HABC concept cannot be implemented rigorously. More precisely, the required equivalent currents are not known on the boundary where they must be applied so that they are replaced with an estimate computed using an operator, for example, a Higdon operator [5], [6]. However, the HABC is not just another implementation of traditional operator ABCs [4]. First, it can be easily designed to absorb evanescent waves [4]. Second, it can be combined with such other ABCs as the PML ABC [4] or a real stretch of coordinates [7]. For these reasons, and since in addition it is simpler than the PML ABC, the $\mathrm{HABC}$ is a promising $\mathrm{ABC}$ that may challenge the well-established PML ABC in some problems, or can be used to improve the effectiveness of other ABCs.

Manuscript received August 4, 2011; revised November 23, 2011; accepted December 20, 2011.

F. Costen is with the School of Electrical and Electronic Engineering, University of Manchester, Manchester M13 9PL, U.K., and also with RIKEN, Saitama 351-0198, Japan (e-mail: f.costen@cs.man.ac.uk).

J.-P. Bérenger is with the Centre d'Analyse de Défense, 94110 Arcueil, France (e-mail: jpberenger@gmail.com).

Digital Object Identifier 10.1109/TEMC.2012.2186302
The papers [1]-[4] mainly present the principle and the theory of the proposed ABCs on a plane boundary assumed as of infinite extent. Although numerical experiments are reported in these papers in two or three dimensions, little attention is paid to the corners of the computational domain. By contrast, this paper focuses precisely on this question, which is a critical question since the equivalent currents that generate the opposite wave are not just a usual Huygens surface.

As stated by the Shelkunoff theorem, a Huygens surface is a closed and continuous surface that splits the physical space into two regions, in general, an interior region and an exterior region. Sources in one region can be replaced with equivalent currents that radiate the same field as the sources into the other region. Huygens surfaces are currently used with the finitedifference time-domain (FDTD) method either to generate an incident wave or for the near-to-far field transformation [8]. In Cartesian coordinates, they take the form of a parallelepiped. In the HABC [4] and in its special cases [1] and [2], [3], the situation is more complex. The reason is that the exact equivalent currents are replaced with estimates computed with an operator that is discontinuous at the corners of the HABC. If a normal Huygens surface is used. the radiated field is discontinuous so that spurious sources are produced in the corners. To overcome this problem, additional surfaces must be added to the Huygens surface. They extend from the corners or edges to the outer end of the computational domain. We call them the extensions of the Huygens surface.

In papers [1] and [2], [3], the need for extensions is not discussed and even mentioned. However, there is some evidence, as in Fig. 6 in [1] or in Table I in [3], that the Huygens planes were extended up to the outer boundary ending the domain. In other words, what we call here extensions were used in the numerical experiments. The rationale for the authors to do this is not reported. It may be a proper understanding of the corner problem or the simplification of the implementation suggested by a brief comment in [1]. Thus, in view of future developments of the HABC, there is a need for clarification and a proper theoretical justification of the implementation in the corners. This is the purpose of this paper. It shows in detail why the extensions are needed and reports some numerical experiments that demonstrate the impact of the extensions on the correctness of the computed results. The absence of extension has a limited impact on the results when the HABC is set closely to the outer boundary ending the domain, as in the experiments in [1]-[4], but when the space between the HABC and the outer boundary is large, as in the method [7], the presence of the extensions is primordial. Without extension, the computed results are strongly erroneous. 


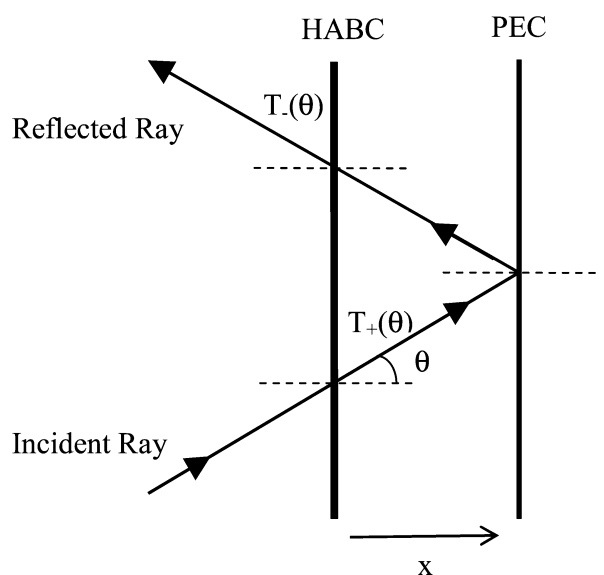

Fig. 1. A plane wave propagating toward an infinite wall HABC. with

$$
T_{+}(\theta)=\frac{1}{1-\sum_{k=M+1}^{N} a_{k}} \sum_{k=1}^{N} a_{k}\left(\frac{\delta t_{k}-\cos \theta \delta x_{k}}{c}\right)
$$

where $c$ is the speed of light. We assume that the numerical 115 technique is the FDTD method [8] so that the shifts in space and 116 time $\delta x_{k}$ and $\delta t_{k}$ are multiples of the steps on space and time 117 $\Delta x$ and $\Delta t$, respectively. We use the first-order Higdon operator 118 with which the estimate reads:

$$
\begin{aligned}
\tilde{U}^{n+1}\left(I_{\mathrm{HABC}}\right)= & U^{n}\left(I_{\mathrm{HABC}}-1\right)++w U^{n+1}\left(I_{\mathrm{HABC}}-1\right) \\
& -w \tilde{U}^{n}\left(I_{\mathrm{HABC}}\right)
\end{aligned}
$$

where $n$ is the index on time, $I$ is the index on space in $x \quad 120$ direction, and

$$
w=\frac{c \Delta t-\Delta x}{c \Delta t+\Delta x}
$$

The estimation (4) is a special case of (1) with $M=2$ and $N=122$ 3 . Using (1), (3), and (4), the coefficient $T_{+}(\theta)$ of the Higdon 123 operator is obtained as follows:

$$
\begin{aligned}
T_{+\mathrm{Hig}}(\theta) & =\frac{1}{1+w}\left[\frac{(1-w) \Delta t-\cos \theta(1+w) \Delta x}{c}\right] \\
& =\frac{(1-\cos \theta) \Delta x}{c} .
\end{aligned}
$$

Consider now a wave $U_{i-}(t)$ propagating from the right-hand 125 side of the HABC, such as the reflected ray in Fig. 1. From [4], 126 the wave $U_{t-}(t)$ transmitted to the left-hand side of the HABC 127 is the integral on time of $U_{i-}(t)$ :

with

$$
T_{-}(\theta)=\frac{1-\sum_{k=M+1}^{N} a_{k}}{\sum_{k=1}^{N} a_{k}\left(\delta t_{k}+\cos \theta \delta x_{k} / c\right)}
$$

or in the special case of the Higdon operator:

$T_{-\mathrm{Hig}}(\theta)=\frac{1+w}{[(1-w) \Delta t+\cos \theta(1+w) \Delta x / c]}=\frac{c}{(1+\cos \theta) \Delta x}$.

For the problem in Fig. 1, the incident wave is differenti- 131 ated by the HABC and transmitted with the coefficient $T_{+}(\theta), \quad 132$ reflected from the PEC with the coefficient -1 , and then in- 133 tegrated by the HABC and transmitted back into the interior 134 domain with the coefficient $T_{-}(\theta)$. The net result is the apparent 135 reflection:

$$
R(\theta)=-T_{-}(\theta) T_{+}(\theta)
$$




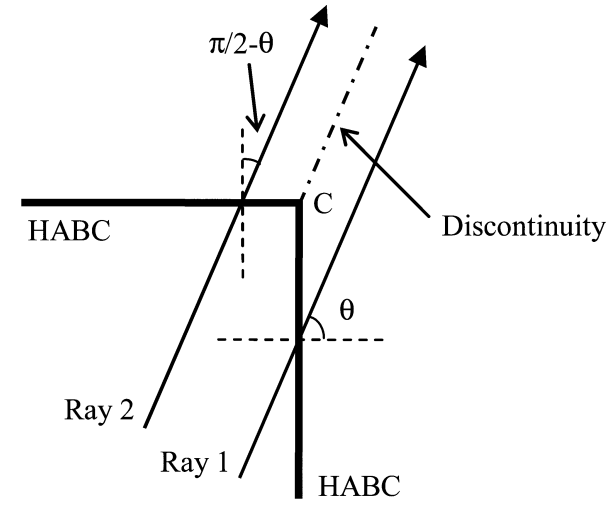

Fig. 2. Discontinuity of the transmitted wave at the corner of a $2 \mathrm{D}$ HABC.

where a term corresponding to the free space propagation between HABC and PEC has been omitted. For pure traveling waves, this term is a phase term of modulus one. For evanescent waves, it would equal the natural decay of the wave. As shown in [4], the reflection (10) equals, rigorously, the reflection obtained by enforcing the estimate (1) as a boundary condition. This is easily verified in the case of the Higdon operator, where using (6), (9), and (10), we obtain $R(\theta)=-(1-\cos \theta) /(1+$ $\cos \theta$ ), which is the well-known reflection from the first-order Higdon operator [5], [6]. Therefore, the ABC composed of a HABC surface and a PEC surface is equivalent, rigorously, to the corresponding operator $\mathrm{ABC}$.

\section{HABC AT THE EDGES AND CORNERS OF A COMPUTATIONAL DOMAIN}

Let us first consider the 2-D case depicted in Fig. 2, where an HABC without PEC behind it radiates the wave opposite to the incident wave. Assume that the outgoing wave is a plane wave at incidence $\theta$ with respect to the vertical in Fig. 2, and consider two rays that strike the $\mathrm{HABC}$ to the right and to the left of the corner $\mathrm{C}$. Then the incidence on the Huygens surface is $\theta$ for ray 1 and $\pi / 2-\theta$ for ray 2 . The transmitted waves corresponding to the two rays are the derivative on time of the incident wave, multiplied with the coefficients $T_{+}(\theta)$ and $T_{+}(\pi / 2-\theta)$, respectively, where $T_{+}$is given by (3) in general or (6) in the special case of the Higdon operator. It is obvious from (3) or (6) that the magnitudes of the two transmitted rays are different, except in the case where $\theta=\pi / 4$. This is true for all rays as long as they strike the HABC on the two sides of the corner $\mathrm{C}$, and whatever may be their distance from $\mathrm{C}$. Thus, the transmitted field is discontinuous behind the HABC. The wave is no longer a plane wave. Its magnitude varies in the direction perpendicular to the propagation. This also occurs in 3-D at the edges of the HABC, which are similar to 2-D corners, and at the 3-D corners, where there are three different transmission coefficients. In both 2- and 3-D, such discontinuities produce additional components to the field, i.e., they act as spurious sources.

The discontinuity in the transmitted wave originates in the estimate of the opposite field radiated by the Huygens surface. If the exact outgoing field was used as equivalent current, the

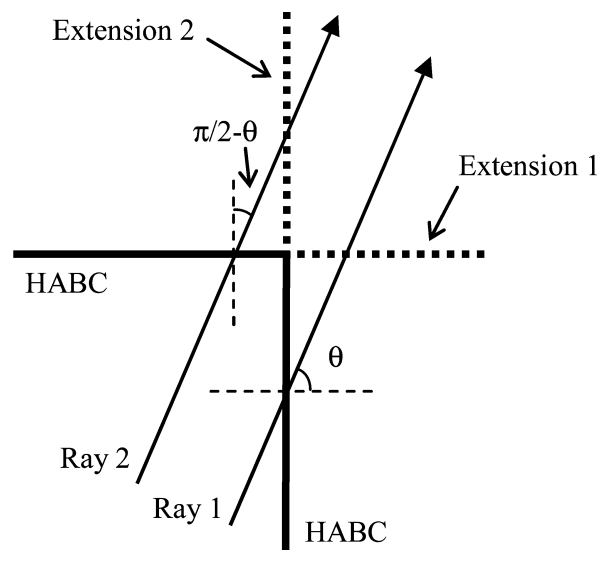

Fig. 3. The extensions of the HABC at the corners in the $2 \mathrm{D}$ case.

radiated field would be exact. With the HABC, the unknown outgoing field is replaced with an estimate computed using an operator that depends on the incidence on the Huygens surface. At the corner, the operator and then the estimates (3) or (6) are discontinuous simply because the incidence angle experiences an abrupt change, from $\theta$ to $\pi / 2-\theta$ in the 2-D case in Fig. 2 . This results in a discontinuity in the radiated field that produces a nonphysical source that may be very large, as demonstrated by a numerical experiment in the Section V.

\section{EXTENDED HABC}

A simple modification of the Huygens surface permits the discontinuity in the transmitted field to be removed. Let us first consider the 2-D case. The modification is depicted in Fig. 3. It consists of extending the HABC surfaces (reduced to lines in 2-D) up to infinity in theory or up to the end of the computational domain in practice. The equivalent currents on the extensions are enforced as on the normal HABC surfaces. For instance, the equivalent currents on extension 1 are computed using the same operator as on the normal horizontal HABC and are enforced by means of the same modifications to the Maxwell equations.

With the extended HABC in Fig. 3, ray 1 crosses the vertical HABC, where it is differentiated on time and transmitted with coefficient $T_{+}(\theta)$. Then it crosses extension 1 , where it is differentiated another time and transmitted with $T_{+}(\pi / 2-\theta)$. Similarly, ray 2 crosses the horizontal HABC, where it is differentiated and transmitted with $T_{+}(\pi / 2-\theta)$, and then crosses extension 2, where it is differentiated and transmitted with $T_{+}(\theta)$. In the region in-between the two extensions, the two transmitted waves are the second derivative of the incident wave and their magnitudes are equal to $T_{+}(\theta) T_{+}(\pi / 2-\theta)$. Thus, in this region, the discontinuity of the field is removed and the field remains a plane wave.

In summary, the space outside the HABC is split into three regions. Two regions, where the field is the first derivative on time, separated by the region, where the field is the second derivative on time. The field is a true plane wave in each region. As verified by numerical experiments in Section V, no spurious source is present. 


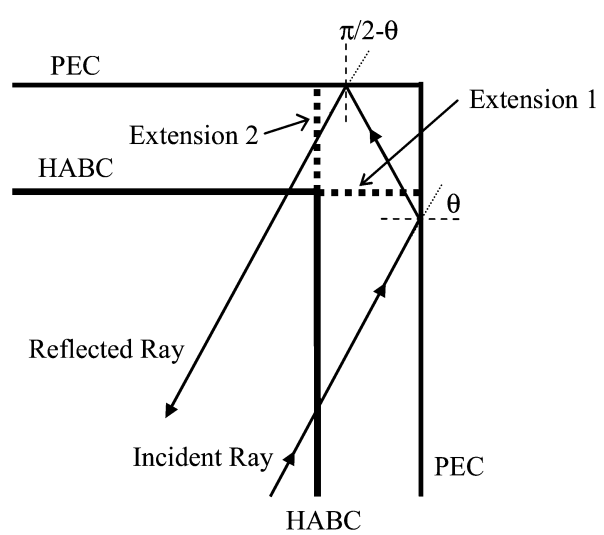

Fig. 4. Reflection of a plane wave from the corner of an extended HABC.

Fig. 3 and the aforementioned derivations address the 2-D case. In 3-D, there are edge regions similar to the 2-D corners. At the 3-D corners, by extending the three Huygens planes up to infinity, the space outside the HABC is split into seven regions. It can be seen that the field in all the regions is a true plane wave, without discontinuity of the transmission coefficient. In three regions, the field is the first derivative of the incident wave, in three others it is the second derivative, and in the last it is the third derivative. In this seventh region, the transmission is the product of three coefficients (3) or (6) and is the same for all the rays so that the continuity of the field is ensured.

Consider now the reflection of a plane wave from the corner region when a PEC is present behind the HABC, as depicted in Fig. 4. The ray shown in Fig. 4 is differentiated and transmitted by the vertical HABC with the coefficient $T_{+}(\theta)$, reflected from the PEC, differentiated and transmitted by the horizontal extension 1 with $T_{+}(\pi / 2-\theta)$, reflected from the horizontal PEC, integrated and transmitted by extension 2 with $T_{-}(\theta)$, and finally integrated and transmitted by the horizontal HABC with $T_{-}(\pi / 2-\theta)$. The net reflection reads:

$$
R_{\text {EXTcorner }}=T_{+}(\theta) T_{+}\left(\frac{\pi}{2-\theta}\right) T_{-}(\theta) T_{-}\left(\frac{\pi}{2-\theta}\right) .
$$

Using (10), this can be rewritten as follows:

$$
R_{\text {EXTcorner }}(\theta)=R_{\mathrm{ABC}}(\theta) R_{\mathrm{ABC}}\left(\frac{\pi}{2-\theta}\right)
$$

where $R_{\mathrm{ABC}}(\theta)$ and $R_{\mathrm{ABC}}(\pi / 2-\theta)$ are the reflections from the operator $\mathrm{ABC}$ on the vertical and horizontal boundaries, respectively. From this, the reflection from the corner of the HABC is identical to the reflection from the corner of the domain bounded by the operator $\mathrm{ABC}$ relying on the same operator as the HABC. Obviously this is not true in the absence of extensions, since then the coefficients $T_{+}(\pi / 2-\theta)$ and $T_{-}(\theta)$ are missing in (11) so that the reflection differs from the reflection from the corner of an operator $\mathrm{ABC}$ (12). The presence of the extensions permits the discontinuity to be removed in the corner, and renders the $\mathrm{HABC}$ equivalent, rigorously, to the corresponding operator $\mathrm{ABC}$.

Fig. 4 addresses the 2-D case. In 3-D, the three Huygens planes are extended up to the PEC. The reflection from the

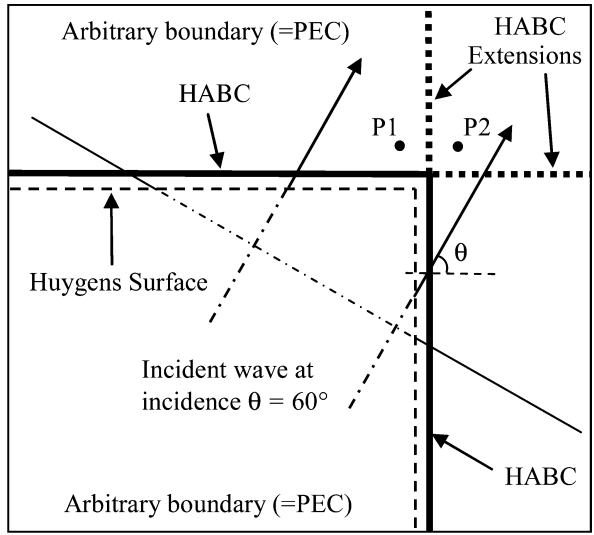

Fig. 5. Numerical experiments with a plane wave striking a corner of the HABC. The spatial and time steps of the 2D FDTD domain are $5 \mathrm{~cm}$ and $100 \mathrm{ps}$, respectively.

$\mathrm{HABC}$ corners is then the product of six coefficients, which 250 is the generalization of (11) to 3-D. As in 2-D, this reflection 251 equals that of the corresponding operator $\mathrm{ABC}$.

The implementation of the HABC extensions in a computer code is simple. Since the equivalent sources on the extensions are identical to those on the normal HABC surfaces, the only thing to do is a change of the limits of the loops, where the equivalent currents are enforced. This is apparently what was done with the rRBC from the Table I in [3].

In principle, the extended $\mathrm{HABC}$ could be used with other outer boundaries than the PEC, so as to combine the HABC with another $\mathrm{ABC}$ [4]. At least with operator $\mathrm{ABCs}$, which use only nodes located in the direction perpendicular to the $\mathrm{ABC}$. This is the case with Higdon operators, but not with Engquist-Majda ABC [9], which uses FDTD nodes in the transverse direction. With the PML ABC, we think that the extension of the HABC is possible as well by using extensions up to the PEC ending the PML.

Only homogeneous waves have been considered previously. However, the results and conclusions in [4] also apply to nonhomogeneous waves. Thus, the derivations and conclusions about the corner regions are also valid for evanescent waves, i.e., the extensions are also needed with evanescent waves. This is illustrated in Section V by an experiment with a scattering structure surrounded with strongly evanescent fields.

\section{NUMERICAL EXPERIMENTS}

This section reports two experiments, which validate the theory in the previous section, and one experiment, which demonstrates that the extension of the HABC is of primordial importance for further developments of the HABC technique. The experiments were performed in the 2-D case, which permits an easy generation of incident plane waves.

\section{A. Continuity of the Field Behind the Extended HABC}

In this experiment depicted in Fig. 5, a plane wave strikes the 283 corner of a 2-D HABC. The wave is generated by means of a 284 Huygens surface [8] placed close to the HABC. Both surfaces 285 


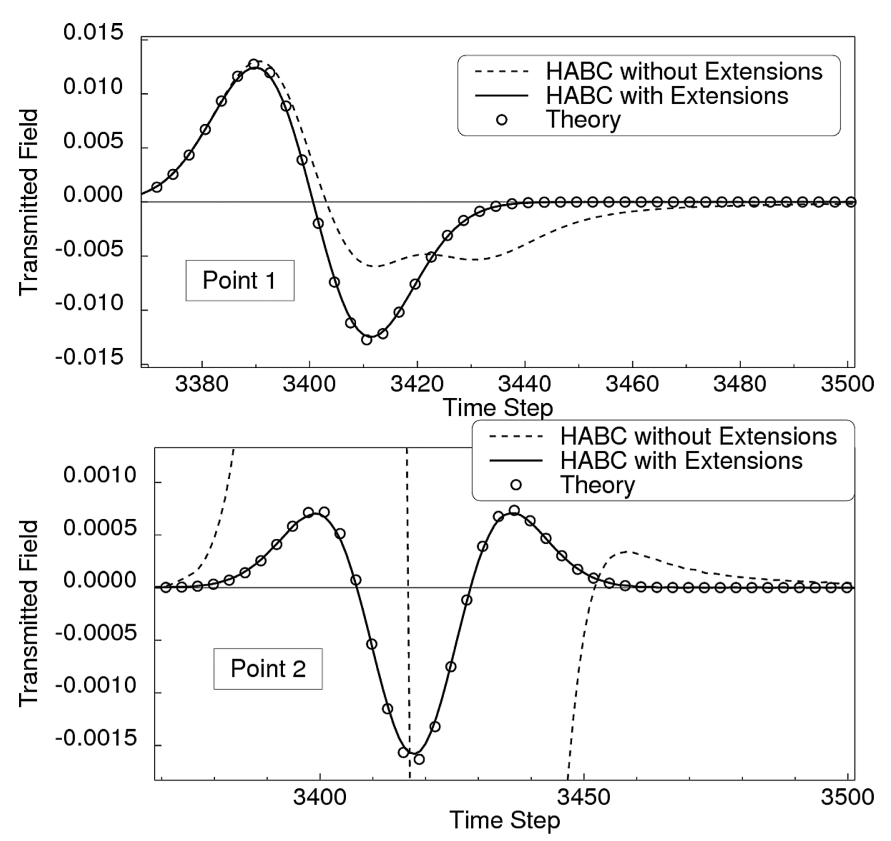

Fig. 6. The field transmitted behind the HABC in the absence of PEC, at P1 (upper part) and P2 (lower part) in Fig. 5. Comparison of the theoretical field with the FDTD fields computed with and without HABC extensions. are truncated by nonphysical conditions so that the calculation is only valid for a clear time which is about 350 time steps at the corner region with the larger than 2000 cells domain used in the experiment. The incidence is $60^{\circ}$ with respect to the vertical ABC. The incident wave is a Gaussian pulse 15 time steps in width. The FDTD domain is large enough behind the HABC in order to ensure that the reflection from its outer boundary is not viewed in the corner region for the whole duration of the calculation.

The calculations have been performed using the HABC with and without extensions. The results are shown in Fig. 6 at the two locations denoted as P1 and P2 in Fig. 5.

At point $\mathrm{P} 1$, the wave should be the derivative of the incident wave multiplied with the coefficient $T_{+\mathrm{Hig}}(\pi / 2-\theta)$. This theoretical prediction is plotted in the upper part of Fig. 6 . The extended HABC result agrees very well with the theory. Without extension, the field differs from the prediction because of the discontinuity of the transmission by the HABC.

At point 2, the field with the extended HABC should be the second derivative of the incident field multiplied with $T_{+}(\pi / 2-$ $\theta$ ) and $T_{-}(\theta)$. The lower part of Fig. 6 compares this theoretical prediction with the FDTD results. The agreement is excellent with the extended HABC. Conversely, without extension, the field is quite different because of the discontinuity and because it remains proportional to the first derivative so that its magnitude is about that at P1.

\section{B. Comparison of the HABC With an Operator $A B C$ in a Corner Region}

In this experiment depicted in Fig. 7, a PEC is present 20 FDTD cells behind the HABC. As in Fig. 5, the incident wave is generated by a Huygens surface close to the HABC so that

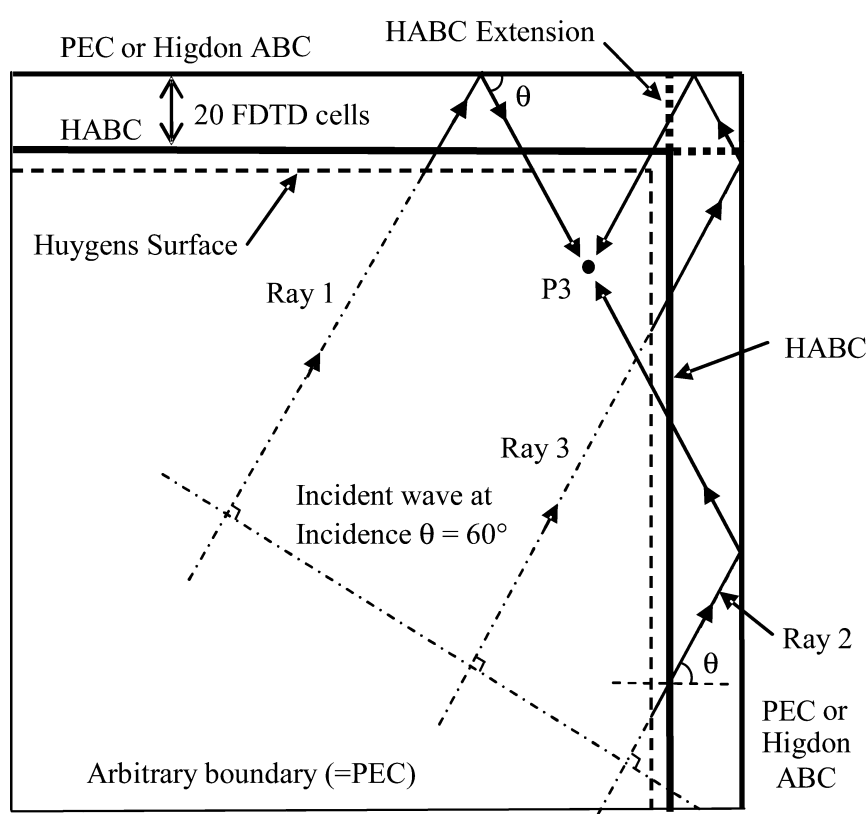

Fig. 7. Numerical experiments with a plane wave striking a corner of the $\mathrm{HABC}$, with a PEC behind the HABC.

TABLE I

The Three Calculations With the Domain In Fig. 7

\begin{tabular}{|c|c|c|c|}
\hline Calculation & Outer boundary & HABC & HABC extension \\
\hline 1 & PEC & yes & no \\
\hline 2 & PEC & yes & yes \\
\hline 3 & Higdon ABC & no & no \\
\hline
\end{tabular}

only the reflected field is present in the interior domain at the observation point $\mathrm{P} 3$. Three calculations were performed whose calculation settings are summarized in Table I. The first one with the PEC and the HABC without extension, the second one with the PEC and the HABC with extensions, and the third one without $\mathrm{HABC}$ and with the PEC replaced with the Higdon operator $\mathrm{ABC}$. The three reflected fields at point $\mathrm{P} 3$ are plotted in Fig. 8, with in addition to the theoretical reflection from a Higdon operator ABC.

We can observe three pulses in Fig. 8. They correspond to the three rays represented in Fig. 7. Ray 2 is the reflection from the vertical boundary with incidence $\theta$, ray 1 from the horizontal boundary with incidence $\pi / 2-\theta$, and ray 3 from the corner. For the Higdon operator $\mathrm{ABC}$ and $\theta=60^{\circ}$, the corresponding reflections $R(\theta), R(\pi / 2-\theta)$, and $R(\theta) R(\pi / 2-\theta)$ are $0.3333,0.0718$, and 0.0239 , respectively. These reflections have been used along with the differences between the ray paths to compute the theoretical reflection plotted in Fig. 8. As observed, the Higdon operator result and the extended $\mathrm{HABC}$ result agree very well with the expected theoretical reflection. The extended HABC is equivalent, rigorously, to the Higdon ABC. Conversely, without extensions, the HABC yields a strongly different result, the reflection from the corner (ray 3, third pulse) is one order of 


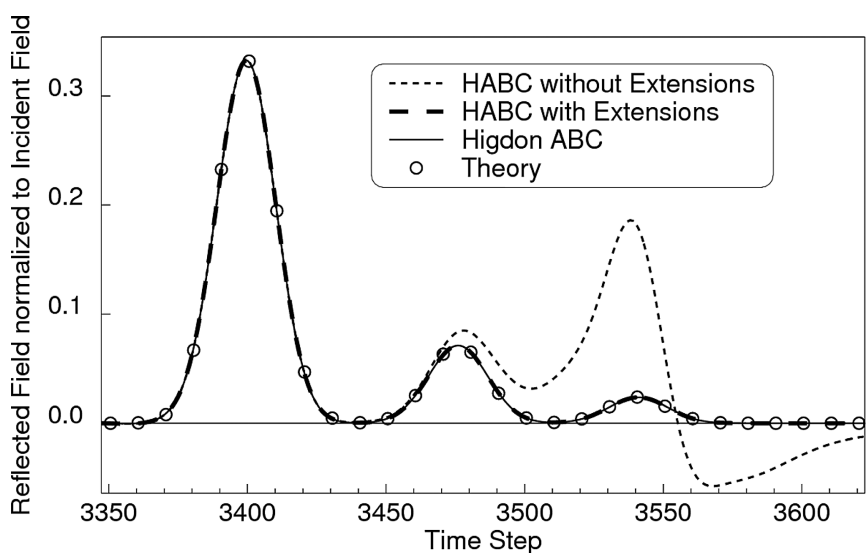

Fig. 8. The reflected field at point $\mathrm{P} 3$ in Fig. 7. Comparison of the theoretical field with the results of the three FDTD calculations defined in the Table I.

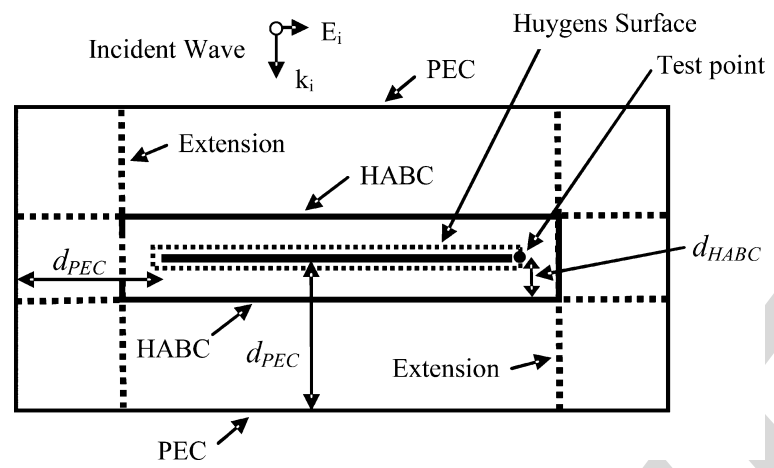

Fig. 9. The 2D FDTD domain for the experiments with a plane wave striking a 2D 300-cell long thin plate. The FDTD steps equal $5 \mathrm{~cm}$ and $100 \mathrm{ps}$. The incident electric field is parallel to the PEC plate and is generated by a normal Huygens surface set 2 FDTD cells from the plate. The HABC is $d_{\mathrm{HABC}}$ from the plate and the outer PEC is $d_{\mathrm{PEC}}$ from the plate.

magnitude larger than the correct reflection, because of the spurious source produced by the discontinuity of the transmitted wave.

\section{Experiment With a Scattering Structure}

A 300-cell-long 2-D PEC plate of zero thickness is struck by an incident plane wave propagating downward (see Fig. 9). The incident waveform is a unit step with a rise time of 10 time steps. An HABC surface and a PEC are placed at $d_{\mathrm{HABC}}$ and $d_{\mathrm{PEC}}$ from the plate, respectively. For the experiments reported in Fig. 10, the HABC surface is placed at various distances $d_{\mathrm{HABC}}$ from the PEC structure, ranging from 10 to 898 FDTD cells, and the PEC is 900 cells from the structure in all the calculations. Fig. 10 shows the electric field normal to the surface at the end of the plate. The HABC without extensions is used in the upper part of Fig. 10, and the extended HABC in the lower part.

As can be observed in Fig. 10, the effect of the extension is very important when the separation between the scattering structure and the HABC becomes small. Even with $d_{\mathrm{HABC}}=$ 10 cells, which is only $1 / 30$ of the structure size, the extended HABC yields results superimposed on the solution computed with the HABC placed 900 cells away, i.e., almost superimposed on the exact solution. This means that the Higdon operator
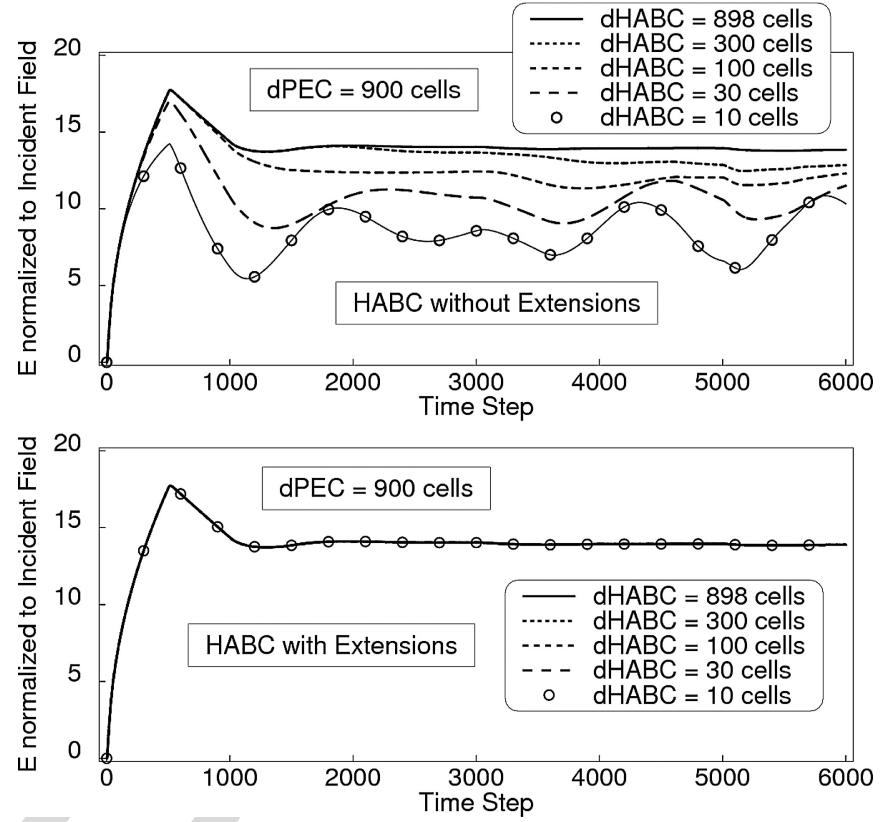

Fig. 10. Comparison of the HABC without extension (upper part) and with the extensions (lower part) when a plane wave strikes a PEC object. The outer $\mathrm{PEC}$ is 900 cells from the object and the HABC is placed various distances from the object.

implemented as an HABC can very well absorb the traveling 362 waves even when it is quite close to the scattering structure. 363 Obviously, the evanescent waves are not absorbed by the HABC 364 based on the Higdon operators [4]. In the experiments in Fig. 9, 365 they decrease in the large space surrounding the HABC so that 366 their apparent reflection is negligible.

\section{EFFECTIVE ABC FOR ELECTROMAGNETIC COMPATIBILITY PROBLEMS}

Typical problems of electromagnetic compatibility consist of 370 computing the field on the surface or in the vicinity of a PEC 371 object struck by an incident wave. In such problems, the scat- 372 tered field is composed of traveling waves at high frequency 373 and evanescent waves at low frequency, with a transition about 374 the fundamental resonance of the object. As shown above, an 375 HABC with the Higdon operator effectively absorbs the trav- 376 eling waves, even if it is close to the object. However, a large 377 domain is still needed outside the HABC for the natural decrease of the evanescent waves.

To reduce the exterior domain, several ways can be imagined. One is the introduction of an operator designed for the evanescent waves, used either as a traditional operator $\mathrm{ABC}$ or in the form of an additional HABC. This was tested successfully in waveguides [4]. Another idea was introduced in [7]. It consists in keeping a large physical domain, as in Fig. 10, but with a strongly stretched FDTD mesh, so as to reduce the overall number of FDTD cells. This is possible because only the low frequency evanescent waves must be absorbed outside the HABC. Since their characteristic length of decrease is of the order of the structure size, use of quite large FDTD cells can be envisaged. This has been confirmed by experiments such as that

\section{.} .

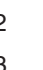

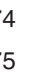

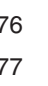



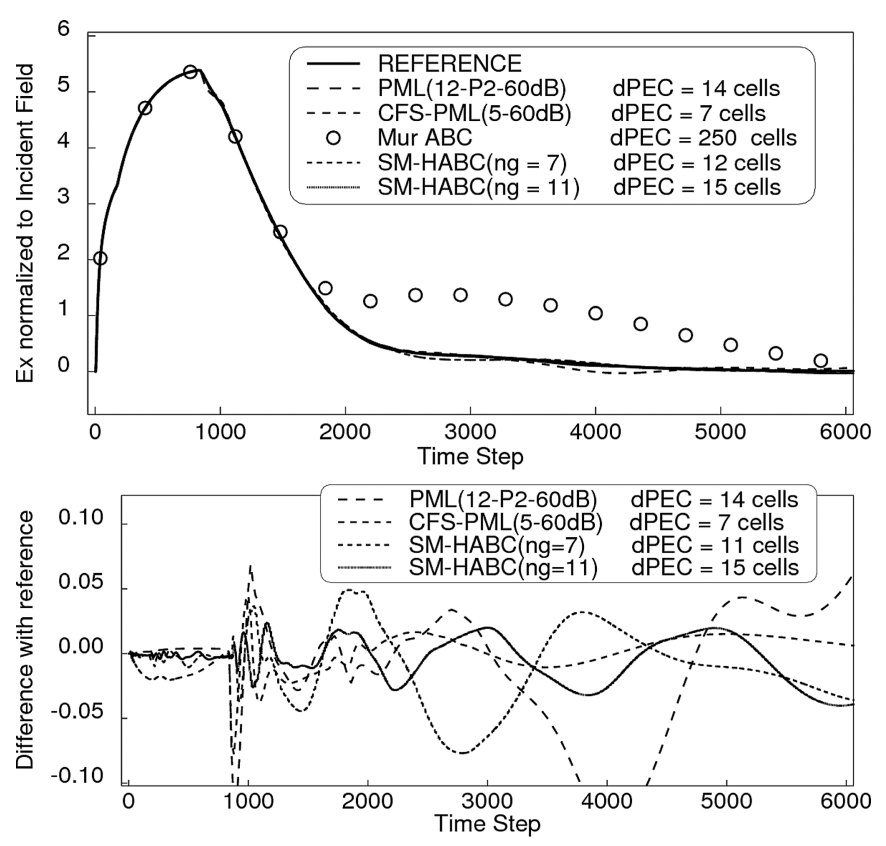

Fig. 11. Electric field at the corner on the surface of a $2 \mathrm{D} 500 \times 50 \mathrm{PEC}$ parallelepiped computed with various absorbing boundary conditions. The lower part is the difference of the computed result with the reference solution.

in [7]. Thus the combination of an HABC for the absorption of the traveling waves with a stretched mesh for the absorption of the evanescent waves forms an effective ABC. We call it the Stretched Mesh HABC (SM-HABC).

A comparison of the SM-HABC with other $\mathrm{ABC}$ s is provided in Fig. 11 with a 2D canonical case. The incident wave is a double exponential of the form $\exp \left(-t / t_{f}\right)-\exp \left(-t / t_{r}\right)$ where $t_{r}=1 \mathrm{~ns}$ and $t_{f}=100 \mathrm{~ns}$. The $2 \mathrm{D}$ object is a $25 \mathrm{~m}$ long parallelepiped of $500 \times 50$ FDTD cells of size $5 \mathrm{~cm}$.

The settings of the SM-HABC calculations are similar to those in Fig. 9, with the $500 \times 50$-cell object surrounded with a HABC placed 3 cells from it, and with a large exterior domain filled with strongly stretched cells. The separation $d_{\mathrm{PEC}}$ between the object and the outer PEC is $75 \mathrm{~m}$, that is 3 object sizes. The stretch of the mesh is a geometrical expansion that begins 4 cells from the object. Two cases are reported in Fig. 11. In the first one, the mesh is stretched upon $n_{g}=7$ cells up to the outer PEC, with ratio $g=2.68$. This means that the separation $d_{\mathrm{PEC}}$, which corresponds to a physical distance of 1500 cells of $5 \mathrm{~cm}$, is filled with only 11 FDTD cells. In the second case, the mesh is stretched upon $n_{g}=11$ cells, with $\mathrm{g}=1.82$, so that the actual separation is $d_{\mathrm{PEC}}=15$ cells.

Two calculations were performed with a PML ABC placed 2 FDTD cells from the object. The first one used a 12 cell thick PML with a polynomial conductivity of power 2 and the normal stretching factor. This PML is not optimum in the sense of [10], but it is probably representative of the PMLs employed by most users. The second one used the best PML which is the CFSPML optimized for 3D wave-structure interactions [11], [12]. It is only 5 cells in thickness. Finally another result in Fig. 11 was computed with the second order Engquist-Majda ABC [9] placed 250 cells from the object.
It is clearly seen in Fig. 11 that the SM-HABC can challenge the PML ABC. Despite the extremely large cells used to fill the large domain, the accordance with the reference solution is similar to the accordance of the PML ABC. More precisely, the lower part of the figure shows that the two SM-HABCs outperform the normal 12-cell PML. Also, the magnitude of the error with the SM-HABC and $n g=11$ cells is very close to that observed with the optimum CFS-PML. The overall number of FDTD cells is slightly larger with the SM-HABC than with the optimum CFS-PML, but the cost of one cell of vacuum is smaller than the cost of one cell of PML. Thus the computational costs of the two ABCs are roughly similar. However, the SM-HABC has a significant advantage in comparison with the CFS-PML. Its implementation is far simpler. This is an attractive feature.

The same conclusions also hold in the 3D case. This will be demonstrated in a forthcoming paper devoted to experiments with realistic $3 \mathrm{D}$ scattering objects.

\section{CONCLUSION}

The implementation of the HABC in the corner regions of 2D or 3D computational domains has been analysed in details. We have shown that the Huygens surface must be extended up to the surrounding PEC. The extensions that may seem in discordance with the equivalence theorem are necessary because of the replacement of the exact outgoing field with an estimate that is discontinuous at the corners of the HABC. The extensions remove the spurious sources produced by the discontinuity, and render the $\mathrm{HABC}$ rigorously equivalent to an operator $\mathrm{ABC}$.

With the extensions the HABC is a highly effective $\mathrm{ABC}$ for the absorption of the travelling waves. By combining the HABC with a strategy to absorb the evanescent waves, as done in this paper with the SM-HABC, highly effective ABCs can be constructed for the solution of problems of electromagnetism, especially in the field of electromagnetic compatibility.

\section{REFERENCES}

[1] I. Wayan Sudiarta, "An absorbing boundary condition for FDTD truncation using multiple absorbing surfaces," IEEE Trans. Antennas Propag., vol. 51, no. 12, pp. 3268-3275, Dec. 2003.

[2] R. E. Diaz and I. Scherbatko, "A simple stackable re-radiating boundary condition (rRBC) for FDTD," IEEE Antenna Propag. Mag., vol. 46, no. 1 , pp. 124-130, Feb. 2004.

[3] R. E. Diaz and I. Scherbatko, "A new multistack radiation boundary condition for FDTD based on self-teleportation of fields," J. Comput. Phys., vol. 203, pp. 176-190, 2005.

[4] J.-P. Bérenger, "On the Huygens absorbing boundary conditions for electromagnetics,” J. Comput. Phys., 2007. doi: 10.1016/j.jcp.2007.04. 008176-190.

[5] R. Higdon, "Absorbing boundary conditions for difference approximations to the multi-dimensional wave equation," Math. Comput., vol. 47 pp. 437-459, 1986.

[6] R. Higdon, "Numerical absorbing boundary conditions for the wave equation," Math. Comput., vol. 49, pp. 65-90, 1987

[7] J.-P. Bérenger and F. Costen, "Application of the Huygens absorbing boundary condition to wave-structure interaction problems," IEEE AP-S Int. Symp., Toronto, Jul. 2010.

[8] A. Taflove and S. Hagness, "Computational electrodynamics: The finitedifference time-domain method," Artech House, 2005.

[9] B. Engquist and A. Majda, "Radiation boundary condition for the numerical simulation of waves," Math. Comput., vol. 31, pp. 629-651, 1977.

[10] J.-P. Bérenger, "Making use of the PML absorbing boundary condition in coupling and scattering FDTD computer codes," IEEE Trans. Electrom. Compat., vol. 45, no. 2, pp. 189-197, May 2003.
441 
[11] J.-P. Bérenger, "Numerical Reflection from FDTD-PML's: A Comparison of the Split PML with the Unsplit and CFS PML's," IEEE Trans. Antennas Propag., vol. 50, no. 3, pp. 258-265, Mar. 2002.

12] J.-P. Bérenger, "The Perfectly Matched Layer for Electromagnetics," Morgan and Claypool, 2007.

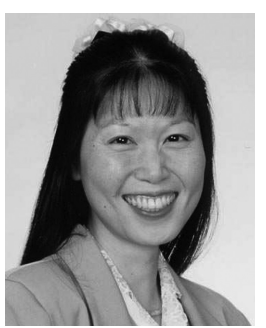

Fumie Costen (M'xx) received the B.Sc. degree, the M.Sc. degree in electrical engineering, and the Ph.D. degree in informatics from Kyoto University, Kyoto, Japan.

From 1993 to 1997, she was with Advanced Telecommunication Research International, Kyoto, where Sshe was engaged in research on direction-ofarrival estimation based on MUSIC algorithm for 3-D laser microvision. She received an academic invitation at Kiruna Division, Swedish Institute of Space Physics, Sweden, in 1996, and gained three patents in 1999 from the research. From 1998 to 2000, she was with Manchester Computing in the University of Manchester, Manchester, U.K., where she was engaged in research on metacomputing, and has been a Lecturer since 2000. Her research interests include computational electromagnetics in such topics as a variety of the finite-difference time-domain (FDTD) methods for LF and high spatial resolution, and FDTD subgridding and boundary conditions. Her work extends to the hardware acceleration of the computation using general-purpose computation on graphics processing units, SSE, and advanced vector extensions instructions

Dr. Costen received an ATR Excellence in Research Award in 1996 and a best paper award from 8th International Conference on High Performance Computing and Networking Europe in 2000.

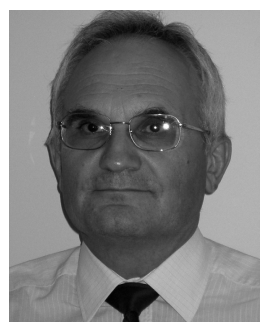

Jean-Pierre Bérenger (F'09) received the Master's 513 degree in physics from the University Joseph Fourier, Grenoble, France, in 1973, and the Master's degree in optical engineering from the Institut d'Optique Graduate School, Paris, France, in 1975.

From 1975 to 1984 , he was with the Département Etudes Théoriques, Centre d'Analyse de Défense, Arcueil, France, where he was engaged in research on the propagation of waves and the coupling problems related to the nuclear electromagnetic pulse. He helped popularize the finite-difference time-domain (FDTD) method in France. In 1984-1989, he was involved in the development of simulation software in the Département Nucléaire. From 1989 to 1998, he held a position as an expert on the electromagnetic effects of nuclear disturbances. He is currently a Contract Manager at the Centre d'Analyse de Défense while staying active in the field of numerical electromagnetics in topics such as LF propagation, absorbing boundary conditions, and FDTD subgridding.

Mr. Bérenger is a member of the Electromagnetics Academy. From 2006 to 2010, he was an Associate Editor of the IEEE TRANSACTIONS ON ANTENNAS AND PROPAGATION.

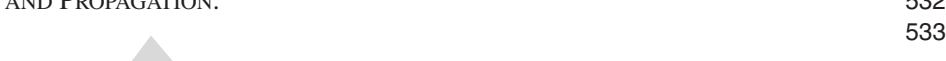

\section{4} 515 
Q1: Author: Please check if the affiliation of author Costen is OK as typeset.

Q2. Author: Please spell out PEC in full at its first occurrence.

Q3. Author: Please provide the years in which author Costen received his B.Sc., M.Sc, and Ph.D. degrees.

Q4. Author: Please spell out MUSIC in full, if possible.

Q5. Author: Please spell out SSE in full, if possible.

Q6. Author: Please provide the title of the Master's degrees of author Bérenger.

Q7. Author: Please check if the second para of author Bérenger's biography is OK as typeset. 